\title{
Liderazgo transformacional en las organizaciones sanitarias. Una revisión bibliográfica
}

\author{
Alina Danet, Inmaculada García Romera, Joan Carles March Cerdà \\ Escuela Andaluza de Salud Pública \\ Instituto de Investigación Biosanitaria de Granada
}

\section{Palabras clave}

Liderazgo.

Gestión organizacional. Sanidad.

\begin{abstract}
Resumen
El objetivo de esta revisión fue conocer los orígenes teóricos, los estilos y modelos de liderazgo transformacional, así como su aplicación y desarrollo en el ámbito sanitario. La revisión bibliográfica se realizó en julio 2013, en las bases de datos electrónicas Scielo, Dialnet Uniroja, Índice Médico Español, PubMed y Ovid. La búsqueda se efectuó en tres fases sucesivas, generando la selección final de 204 documentos, que se estudiaron a través de un análisis de contenido que incluyó: objetivo, abordaje teórico, metodología, resultados, conclusiones. La bibliografía sitúa el origen del liderazgo transformacional en el contexto de una mayor competitividad, cambio e innovación organizacional. A partir de finales del siglo $X X$, surgió un amplio espectro de perspectivas explicativas sobre los diferentes estilos y modelos de liderazgo transformacional, dentro de los cuales destaca especialmente el liderazgo distribuido. En el marco de la organización sanitaria, la línea de innovación organizacional se corresponde con exigencias específicas de liderazgo transformacional y las principales líneas de investigación son: la relación del liderazgo con el clima y la satisfacción profesional, el liderazgo enfermero y la formación en el liderazgo. Nuevos estudios son necesarios para conocer las mejores prácticas y estrategias de liderazgo y gestión en el ámbito de la salud.
\end{abstract}




\section{Transformational leadership in the health care organizations. A bibliographical review}

\section{Key words}

Leadership.

Organizational management. Health care.

Abstract

The objective of this revision is to know the origins, styles and modes of transformational leadership and, in specific way, its application and development in the health care context. The bibliographical review was made during July 2013, using the following databases: Scielo, Dialnet Uniroja, Índice Médico Español, PubMed and Ovid. Successive phases of the search led to 204 documents, which were studied through content analysis, including: objective, theory, methods, results and conclusion. Bibliographical sources Organizational competitivity, change and innovation represent the context for the origins of the transformational leadership. From the end of the 20th century, new explicative perspectives arose about different styles and models of transformational leadership, with special attention to the distributive leadership. The research on health organization responded to specific transformational leadership applied to health innovation and centred on: the relationship between leadership and professional climate and satisfaction, nursing leadership and training. Additional studies are required to better understand the best leadership practices and strategies in the health care field.

Cómo citar el artículo

Danet, A.; García Romera, I.; March Cerdà, J. C. (2016). Liderazgo transformacional en las organizaciones sanitarias. Una revisión bibliográfica. Revista de Comunicación y Salud, Vol. 6, pp. 83-97.

DOI: http://doi.org/10.35669/revistadecomunicacionysalud.2016.6.83-97 
1. Introducción

El interés de las ciencias sociales por analizar el liderazgo en el ámbito organizacional ha surgido a principios del siglo $\mathrm{XX}$, pero fue especialmente a partir de los años treinta cuando los primeros psicólogos industriales se centraron en estudiar las características y rasgos de personalidad del líder (House y Aditya, 1997). Si bien en las décadas de los cincuenta y sesenta, se fueron elaborando diversas teorías sobre aspectos conductuales, situacionales o relacionales del liderazgo (Fernández, 2006), fueron los años ochenta, el escenario de una nueva fase de entusiasmo por los estudios sobre liderazgo, momento en el cual proliferaron las investigaciones sobre nuevos tipos y estilos de liderazgo (Gil et al, 2011), tendencia que podemos identificar hasta el presente.

El concepto de nuevos modelos de liderazgo transformacional, referido a aquellos procesos de liderazgo que inspiran y motivan a los miembros del grupo hasta la superación de sus propias expectativas de trabajo, se fue insertando en el contexto socio-económico y de gestión organizacional que empezó a priorizar la calidad de las organizaciones, como uno de los factores claves de competitividad y diferenciación a nivel organizacional y empresarial (Fariña y González, 1998).

De forma específica, en las últimas dos décadas, el ámbito sanitario ha registrado el surgimiento de una verdadera cultura de calidad, enmarcada dentro de una compleja interrelación de normas y estructuras organizativas, conocimientos y valores profesionales y una gestión general y clínica adecuada (Freire, 2011). Siguiendo a Tresserras i Gaju (2008), la calidad sanitaria supone un esfuerzo por unir la planificación para la salud y la planificación de servicios, que plantea la necesidad de continua adaptación de las instituciones sanitarias, tanto en relación a los recursos humanos como económicos (Büchler, Martin y Büchler, 2006; Teixidor, 2004). Estas nuevas exigencias requieren nuevos desafíos organizacionales y de gestión, entre los cuales Del Llano (2002) lista la economía de contratos, delegación de funciones, descentralización administrativa, sistema de incentivos, reingeniería de procesos o los cambios en los sistemas de información.

En este panorama de gestión sanitaria, el liderazgo transformacional se ha integrado como una dimensión que acepta y promueve la necesidad de cambio y mejora continua de la organización, busca la participación y consenso entre todas las actividades y personas, a la vez que se dirige hacia la consecución de un buen servicio y de la satisfacción de profesionales, pacientes y usuarios y a la adecuación del sistema organizacional sanitario a las dinámicas del cambio social (Trice y Beyer, 1993; Koch, 1994).

Por tanto, frente al paradigma de dirección que ponía el acento en la visibilidad y protagonismo del líder, a partir de los años finales del siglo $\mathrm{XX}$, las ciencias sociales priorizaron el estudio de la figura y roles de un líder transformacional, que, desde sus conocimientos, habilidades y cualidades morales, aspira a liberar el talento y energía de su equipo, tiene la capacidad de centrarse en los demás y ponerse al servicio de la sociedad (Alvarez de Mon, 2006). Sin embargo, la amplia diversidad de posturas explicativas, así como la complejidad intrínseca del concepto y su aplicación en el contexto organizacional, ha generado múltiples definiciones y usos del término.

La utilidad del presente trabajo de revisión bibliográfica responde a la necesidad de englobar la heterogeneidad de criterios e interpretaciones científicas existentes en el estudio sobre el liderazgo transformacional. 


\section{Objetivo}

El objetivo de esta revisión bibliográfica es conocer los orígenes y estilos o modelos de liderazgo transformacional, así como su aplicación y desarrollo en el ámbito sanitario.

\section{Metodología}

La revisión bibliográfica se realizó durante el mes de julio de 2013, en las siguientes bases de datos electrónicas: Scielo, Dialnet Uniroja, Índice Médico Español, PubMed y Ovid, usando los descriptores en español y en inglés: "liderazgo", "leadership", "organización sanitaria", "health organization", "médico", "physician", "enfermería", "nursing".

La búsqueda generó más de 35000 documentos, de los cuales, en una segunda ronda de selección, se incluyeron en una base de datos del software Mendeley, sólo los materiales que cumplieron los siguientes criterios de inclusión: 1. Tener fecha de publicación posterior a 1980; 2. Ser publicado en revistas indexadas, con o sin factor de impacto (criterio Journal Citation Report); 3. Incluir en el título, resumen o cuerpo el término "liderazgo transformacional" o "transformational leadership".

Esta selección generó un total de 529, que se analizaron de forma descriptiva a partir de su título y resumen, prestando atención a las siguientes variables: objetivos, metodología y concepto/estilo de liderazgo transformacional.

En una tercera fase de selección se incluyeron sólo aquellos documentos centrados en las siguientes temáticas: orígenes teóricos del liderazgo transformacional, estilos y tipos de liderazgo transformacional y el liderazgo transformacional en el ámbito sanitario. Para los 204 documentos seleccionados se realizó un análisis de contenido que incluyó: objetivo, abordaje teórico, metodología, resultados, conclusiones.

\section{Resultado}

Orígenes teóricos el liderazgo transformacional: los años ochenta.

Según explican Gil y sus colaboradores (2011), el proceso de globalización, los cambios económicos y sociales y la incorporación de nuevas tecnologías de la comunicación generaron, a partir de los años ochenta, cierta preocupación entre los investigadores sociales sobre cómo los dirigentes podían transformar y revitalizar las organizaciones. La necesidad de derribar las pesadas culturas burocráticas, en un entorno organizacional cada vez más competitivo, caracterizado por la existencia de equipos multiprofesionales y múltiples diferencias culturales (Kaufmann, 1997), hacía inminente también a nivel teórico superar el enfoque bivalente que había caracterizado las décadas anteriores y que polarizaba la labor del líder, en base a un carácter instrumental -centrado en los objetivos, tareas, procedimientos y reparto de funciones-, y otro de índole social -orientado a mantener un buen clima, fortalecer la cohesión y la satisfacción de las personas (Torrado, 2012).

A partir de la ruptura de este modelo bipolar, el concepto de liderazgo se integró en un escenario organizacional que daba prioridad al cambio continuo, la necesidad de formar a personas expertas en habilidades dirigentes y apostar por una mayor improvisación, actualización y razonabilidad. Siguiendo a Avolio, Walumbwa y Weber (2009), Gil et al (2011) apuntan que, para responder a una dinámica social cada vez más compleja, los nuevos modelos de liderazgo se fueron dirigiendo, desde los últimos años del siglo XX y hasta la actualidad, hacia un modelo diádico, compartido, relacional, estratégico y global. En la misma línea argumental, también Yukl (2004) explica cómo, a la responsabilidad del líder de conseguir las metas del equipo y mantener la organización en un funcionamiento viable, se le sumó de forma irrevocable la necesidad de propiciar el cambio y la innovación. 
modelo explicativo sobre las funciones del liderazgo fue migrando, según este autor, hacia un sistema triangular, que prioriza no sólo las tareas, sino también las relaciones y el cambio.

Por tanto, en este contexto organizacional actual, donde la promoción de la innovación representa la línea estrategia clave para asegurar la supervivencia y la competitividad (Pons y Ramos, 2011), la investigación sobre liderazgo(s) se focalizó, tanto en el ámbito científico como en el profesional, en su función para desarrollar y poner en práctica la innovación (Janssen, De Vliert y West, 2004).

Es en este marco donde surgieron las aportaciones teóricas sobre el liderazgo transformacional, cuyos orígenes se pueden situar en las teorías de Burns (1978) y Bass (1985), que explicaron el estilo de liderazgo transformacional, a partir de la diferenciación con el estilo transaccional.

Según estos autores, la principal diferencia entre ambos enfoques de liderazgo se sitúa en la cultura organizativa y la gestión de recursos humanos. En el estilo transaccional, el liderazgo representa un proceso de intercambio de mutuo interés entre líderes y sus seguidores, en el cual el dirigente promueve la estabilidad y continuidad en la organización, en base a una recompensa contingente (el líder reconoce la labor exitosa de los subordinados, especificando claramente la recompensa) y a la dirección por excepción (el líder interviene para corregir o castigar a sus colaboradores ante la existencia de un problema, bien de forma preventiva - dirección por excepción activa, bien a posteriori dirección por excepción pasiva). Por otra parte, el liderazgo transformacional se orienta hacia el cambio organizativo y la motivación de los colaboradores para actuar más allá de sus propias expectativas, para conseguir así un alto rendimiento y un elevado grado de implicación en el grupo (Gil et al, 2011). Bass (1985) detalla el mecanismo del liderazgo transformacional, a partir de la confluencia de cuatro elementos básicos: 1. influencia idealizada (a través de la cual se evoca una visión de la organización y se gana la confianza del equipo); 2. motivación inspiradora (es el proceso a través del cual se comunica y transmite la visión compartida y el espíritu de grupo); 3. estimulación intelectual (una forma de promover la solución de problemas y la innovación) y 4. consideración individualizada (trato cuidadoso y adaptado a cada persona).

Algunos autores, como por ejemplo Salas Vallina (2013), consideran que el liderazgo transformacional surge de forma posterior y como un elemento opuesto al estilo transaccional. Sin embargo, otros investigadores no aceptan el carácter independiente y correlativo de estos dos modelos (Arredondo, 2002), encontrando incluso elementos de innovación (Keeley, 1998) o de integridad y reciprocidad superior-subordinado en el modelo transaccional (Aronson, 2001).

El modelo teórico desarrollado por Bass en 1985, posteriormente revisado y ampliado, impulsó a partir de los años finales del siglo XX y en los comienzos del siglo XXI, numerosas aportaciones teóricas y aplicaciones prácticas del modelo (Burke y Cooper, 2006). El liderazgo transformacional fue objeto de revisión de numerosas meta-análisis, entre las cuales Gil et al. (211) destacan las más recientes de Harms y Crede (2010) o Judge y Piccolo (2004).

Estilos y tipos de liderazgo transformacional

La bibliografía disponible recoge diferentes criterios en base a los cuales organizar los modelos teóricos sobre liderazgo transformacional. 
A nivel internacional, el trabajo de Conger y Kanungo (1998) destaca tres enfoques explicativos para el liderazgo transformacional: 1. las ya referidas aportaciones de Bass y Avolio; 2. el modelo de Shamir, House y Arthur (1993), que reflejan como la influencia del líder sobre el sentido de identidad de los seguidores vincula a éstos a las metas y a la visión organizativa; y 3. la teoría visionaria de Sashkin (1998), que subraya la capacidad de sacrificio del líder transformacional de anteponer los intereses grupales, con el objetivo de promover una visión organizacional compartida. La importancia de estas tres corrientes viene así mismo confirmada en la literatura de especialidad española, como por ejemplo en el trabajo de Molero Alonso (2002).

Por otra parte, en el ámbito nacional español, Rico et al. (2010) afirman que en los últimos años, la investigación sobre liderazgo transformacional se ha consolidado en relación a tres elementos: 1. liderazgo externo, basado en una figura de líder externo al equipo, sobre el que pivota el rendimiento del grupo (Stewart, 2006); 2. coaching, en el cual el líder se relaciona directamente con el equipo para coordinar los recursos colectivos (Hackman y Wagerman, 2005); y 3. liderazgo compartido o distribuido, que parte de un concepto de liderazgo como una propiedad emergente de la distribución de las funciones de liderazgo entre múltiples miembros del equipo (Carson, Tesluk y Marrone, 2007).

Coincidiendo en parte con este enfoque, otra revisión también desarrollada en el ámbito español (Gil et al, 2011) organiza los variados estilos del liderazgo transformacional en distintos paradigmas: liderazgo de equipo (dentro del cual incluyen el coaching), liderazgo complejo y liderazgo transcultural. Dentro del liderazgo de equipo, Zaccaro, Rittman y Marks (2001) plantean los procesos cognitivos, motivacionales, afectivos y de coordinación como puntos de influencia en el rendimiento del equipo; y Morgueson, Scott y Karam (2010) se ocupan del origen interno y externo del liderazgo. En la teoría del liderazgo complejo, el proceso directivo se revela como un sistema adaptativo y complejo de agentes dinámicos insertados en redes complejas de retroalimentación, que puede tomar la forma de un liderazgo administrativo, facilitador o adaptativo (Marion y Uhl-Bien, 2007). Por último, el liderazgo transcultural incorpora el papel de la cultura en la eficacia del liderazgo, centrándose tanto en las dimensiones culturales que marcan los atributos de liderazgo, como en las creencias compartidas en diferentes culturas sobre los líderes eficaces (Chocar, Brodbeck y House, 2007) o en la actuación de los líderes en diferentes culturas (Elenkov y Manev, 2009).

Por su parte, Salas Vallina (2013), Peiró y Rodríguez (2008) o Pons y Ramos (2012) centran su revisión en la relación del liderazgo transformacional con ciertos elementos de la salud organizacional, presentando la influencia positiva de estos estilos de liderazgo en la satisfacción laboral (Wageman, 2001), la eficacia del equipo (Stocker et al, 2001) o el bienestar de los empleados (Densten, 2005; Kelloway et al, 2002), a la vez que su peso en reducir el estrés (Sosik y Godshalk, 2000), fomentar el aprendizaje organizativo (Senge, 1990) y la creatividad de los empleados (Amabile y Gryskiewicz, 1989). En la bibliografía internacional, también Linley, Joseph y Seligman (2004) destacan la importancia del liderazgo transformacional como generador de una mayor confianza en la gestión, mayor compromiso y sensación de pertenencia por parte del equipo de trabajo, asegurando mayor satisfacción laboral, bienestar psicológico, seguridad física, percepción de autoeficacia, proactividad y otras oportunidades de crecimientos.

Así mismo, las ventajas asociadas a las prácticas del liderazgo transformacional, ha favorecido el desarrollo de la ética en las organizaciones. En este sentido, en la bibliografía 
internacional encontramos la distinción entre liderazgo transformacional auténtico, en el que el líder orienta su trabajo hacia el beneficio de sus colaboradores, llegando a sacrificar sus intereses por el bien colectivo (Michie y Gootie, 2005), y el pseudo-liderazgo, que se guía por los intereses del propio líder (Gil et al, 2011).

En el ámbito hispano, Moreno Pérez (2004) esquematiza la autenticidad del liderazgo transformacional, en base al comportamiento de "las siete C" (carácter, coraje, credibilidad, comunicación, conocimiento, compromiso y comprensión), orientado por la actitud de "las tres H" (humildad, honestidad y humor). Bolívar (2006) recalca que, para liderar, es básico transmitir confianza y sinceridad, cualidades que sólo emergen de la autenticidad.

De las reflexiones teóricas sobre la ética en las organizaciones, surgieron nuevos conceptos y modelos de liderazgo. Así, por ejemplo, encontramos el liderazgo espiritual (Sanders, 1995), el liderazgo sirviente (Greenleaf, 1997), el libertazgo (González, Beltri y Rubi, 2005), también llamado liderazgo de libertades o el liderazgo remoto (Huang, Kahai y Jestice, 2010), presente en los equipos virtuales.

Pese a la variedad de perspectivas y modelos dentro del liderazgo transformacional, quizás uno de los paradigmas más conocido es el liderazgo compartido (Yukl, 1998). También denominado liderazgo distribuido, colaborativo, colectivo o de iguales (Spillane y Diamond, 2007), este modelo destaca la remodelación de tareas y el cambio en la cultura organizativa desde el compromiso e implicación de todos los miembros del equipo. Así, el ejercicio de dirección se configura como un proceso distribuido, compartido entre el conjunto de la organización (Bennet et al, 2003) y se define como "un proceso dinámico e interactivo" (Pearce y Conger, 2003), que surge como una red de influencia mutua que genera la distribución de acciones de liderazgo en el equipo (Day, Gron y Salas, 2004).

En este sentido, el liderazgo distribuido se puede integrar dentro de los nuevos modelos de liderazgo de equipos, a la vez que dentro de los liderazgos complejos, ya que se genera como fenómeno sistémico, grupal y relacional, en el que intervienen varios elementos, miembros y circunstancias que demandan múltiples conductas, competencias y roles de liderazgo (Gil et al, 2011).

Pese a que algunos autores consideran que este modelo ha sido aún insuficientemente investigado (Rico et al, 2010), desde las ciencias sociales se han elaborado una amplia gama de recursos bibliográficos, mayoritariamente centrados en la influencia del liderazgo distribuido en la eficiencia y rendimiento de trabajo o la satisfacción laboral de los miembros del equipo. Carson y sus colaboradores (2007) o Ensley, Hmielesky y Pierce (2006) señalan la relación positiva entre el liderazgo compartido y la eficacia de tarea de los equipos, aunque la evidencia empírica no siempre revela este resultado (Mehra et al, 2006). Fernández (2006) apuesta por el liderazgo distribuido como la forma más eficiente de liderazgo en el contexto organizacional actual y Peiró y Rodríguez (2008) sitúan este modelo como una fórmula adaptada al trabajo complejo, interdependiente y de gestión del conocimiento que caracterizan actualmente las organizaciones.

Por tanto, la investigación reciente sobre los nuevos modelos de liderazgo en el ámbito organizacional otorga a los líderes un papel destacado como arquitectos sociales, orientados hacia nuevas identidades organizacionales, cuyas funciones básicas son adaptarse, creando climas de confianza y aprendizaje, así como construyendo una visión de futuro (Heifetz, 1994).

El liderazgo transformacional en los equipos sanitarios 
Con respecto a la constante situación de cambio organizacional que caracteriza el ámbito sanitario, en la bibliografía analizada destaca, en primer lugar, el consenso de los autores acerca de la necesidad de adaptar las estructuras de liderazgo a los nuevos retos organizacionales en salud. Esta exigencia, acompañada por el cada vez mayor interés de los profesionales clínicos por la cultura de cambio organizacional, requiere la alteración de roles y responsabilidades (Del Llano, 2002), la construcción de un nuevo profesionalismo médico así como nuevas estrategias de planificación, ordenación y fidelización de los profesionales (García y Amaya, 2007).

En esta dirección estratégica, autores como Hoyos, Cardona y Correa (2008) señalan la necesidad de integrar las prácticas de liderazgo en un proceso de humanización en salud, caracterizado por incentivar la comunicación y resolución de conflictos, generar cambios en los comportamientos y actitudes de las personas, y promover el bienestar integral de profesionales y pacientes.

Otra línea argumental señala la necesidad de promover un liderazgo participativo y persuasivo, centrado en crear un ambiente de confianza en el que los valores que predominen sean la responsabilidad personal, la ayuda y la colaboración entre los profesionales, el fomento de relaciones cordiales interpersonales, el trabajo en equipo, la creatividad, la confianza, la credibilidad y la consecución de retos. Coincidiendo con las opiniones de Teixidor i Freixa (2004) y Cantera y Yáñez (2008) que destacan el papel del liderazgo en establecer buenos ambientes laborales y adaptar su estilo a las características psicológicas de cada persona, también Menárguez Puche y Saturno Hernández (1998) describen la función clave de los líderes para orientar y dar confianza a los profesionales en un mejor desarrollo de sus actividades.

Para Ruiz López y Carrasco González (2010), la creación de un entorno en el que la excelencia en la asistencia florezca y se estimule la mejora continua, debe contribuir a crear y mantener una cultura de seguridad y, entre las capacidades fundamentales que tienen que desarrollar para ser eficaces, destacan la habilidad de comunicar, planificar, anticipar, prevenir y actuar de acuerdo con principios de justicia y equidad.

Ante la complejidad de perspectivas y enfoques de estudio sobre el liderazgo transformacional en el ámbito sanitario, en la bibliografía consultada encontramos tres líneas de investigación consolidadas, que se van a detallar a continuación.

A. La primera línea de investigación estudia las relaciones entre liderazgo y ambiente de trabajo, clima organizacional y satisfacción de los profesionales. Ekvall y Arvonen (1999) señalan que la conducta del líder influye en todos los aspectos del clima y cuantifican hasta un $65 \%$ la influencia del líder en las variaciones ocurridas en el ambiente de trabajo. El trabajo realizado por Chiang, Salazar y Martín (2011) en hospitales chilenos encuentra que un abordaje relacional directo y estrecho por parte de los responsables, genera un clima de apoyo e innovación estable. También en atención primaria, esta vez en el ámbito nacional, Morales y Molero (1995) y Alcázar, Otero e Iglesias (1996) destacan el liderazgo como condicionante básico del espíritu real de equipo, así como para conseguir el compromiso personal de todos los profesionales con los objetivos de la unidad sanitaria. También Peiró et al (2001), en un estudio con profesionales del sistema primario de salud, encuentran que la conducta de iniciación de estructura de los líderes determina una influencia positiva sobre la satisfacción laboral y un efecto indirecto sobre la tensión laboral mediado por la reducción de las percepciones de ambigüedad de rol. En la misma línea, los mismos autores (1996) destacan que el liderazgo transformacional presenta correlaciones significativas con las 
facetas de la satisfacción laboral. Y según Robles-García et al. (2005) el liderazgo influye en la satisfacción profesional, sobre todo en relación con la calidad del trato con el superior y con la autosatisfacción con el trabajo realizado.

B. En segundo lugar, podemos observar una preocupación creciente por parte de las ciencias sociales hacia las necesidades específicas de liderazgo, manifestadas por el personal sanitario enfermero, así como a los estilos de liderazgo ejercido por parte de esta categoría profesional. Partiendo de su necesidad por aumentar su autonomía laboral y recibir mayor reconocimiento profesional (Martin et al, 2008), especialmente en la relación con el personal facultativo (Subirana y Fargues, 2005), la centralidad de las demandas y expectativas del personal de enfermería rompe con la dicotomía tradicional que situaba a los profesionales de enfermería en una posición de subordinación con respecto a al modelo de hegemonía médica (Carrasco, Márquez y Arenas, 2005).

Precisamente, una de las estrategias que se proponen superar las dificultades que puede encontrar la categoría profesional de enfermería en su medio laboral, es un estilo de liderazgo motivacional, orientado a prevenir el aislamiento y conferir mayor apoyo (Goetz et al, 2012). Algunos autores señalan la importancia de desarrollar las habilidades relacionales de los directivos, como forma de promover valores como el universalismo, logro, tradición y autodirección (Aguilar, Calvo y García, 2007). Sin embargo, otros apuestan por compatibilizar las acciones relacionadas con el trabajo y las personas, pero desde una dinámica participativa, promoviendo valores como la responsabilidad, integridad y compromiso, habilidades de comunicación efectiva, trabajo en equipo y capacidad de gestión y conocimientos específicos para la realización de las tareas y cumplimiento de los objetivos (Angel, 2006).

Asumiendo la importancia del liderazgo formal en la profesión de enfermería (Peya, 2008), De Lima et al (2011) identifican cierta dificultad de esta categoría por comprender el liderazgo, así como su aplicabilidad. También Souza et al (2013) reflejan que, desde la enfermería, existe una conciencia clara sobre la importancia de la relación entre liderazgo y enfermería, así como acerca de la dificultad de ejercer el liderazgo en el equipo multidisciplinar, donde confluyen necesidades específicas dependiendo del área de actuación y de la categoría profesional.

Ante esta limitación, los autores proponen la necesidad de construir espacios donde la enfermería pueda conocer y desarrollar sus habilidades para interpretar y adoptar estilos de liderazgo que mejoren el trabajo en equipo y el cuidado prestado a los usuarios del servicio. Según Lauzon, Ricard y Goulet (2003) el desarrollo de un fuerte liderazgo enfermero, basado en la adquisición de poder colectivo, la búsqueda de la cohesión dentro de la profesión y un profundo compromiso con el avance de la disciplina, son obligaciones para los profesionales de enfermería comprometidos.

C. Liderazgo y formación. La formación de los líderes constituye una tercera línea de investigación actual. Para Büchler, Martin y Büchler (2006), el objetivo de formar en el liderazgo requiere un programa formativo en gestión empresarial durante la etapa académica, a lo que se le tendría que sumar la formación continua a largo de la vida laboral (Morán y Ruiz, 2010). Entendida en el contexto organizacional de continuo cambio e innovación sanitaria -que requiere la adquisición constante de nuevas competencias profesionales-, la formación se contempla cada vez más como una parte integrante del proceso de aprendizaje que los sistemas sanitarios deben desarrollar tanto de manera interna como externa. Así, Córdoba, García Vázquez y Moreno (2011) consideran como 
elementos facilitadores del aprendizaje en la organización la apuesta por las tecnologías de la información y las comunicaciones, la política de recursos humanos, la promoción de un buen ambiente o clima de trabajo, la estructura o diseño organizativo y la cultura organizacional. Entre estos factores de aprendizaje, el liderazgo transformacional, participativo y distribuido aparece como un medio esencial para el desarrollo de nuevas formas de enfocar y entender el aprendizaje organizacional en el seno de la sociedad.

\section{Conclusiones}

La bibliografía disponible sitúa el origen del liderazgo transformacional en los años ochenta, en el contexto de una mayor competitividad organizacional. La nueva cultura de cambio e innovación impusieron, según refleja la literatura disponible, la necesidad de enfocar las funciones de liderazgo, no sólo hacia la consecución de objetivos y tareas, sino también hacia los aspectos relacionales y de cambio organizacional.

A partir de la creciente atención de las ciencias sociales hacia los procesos de liderazgo, a partir de finales del siglo XX, emergieron nuevas perspectivas explicativas sobre los diferentes estilos y modelos de liderazgo transformacional. Ante la variedad de categorías y corrientes explicativas, podemos concluir que la literatura de especialidad se aleja de una explicación unidimensional sobre el liderazgo transformacional unitario, para abrir una amplia gama de liderazgos transformacionales, entre los cuales podemos listar los liderazgos de equipo, complejo, transcultural, coaching, auténtico, espiritual o el conocido liderazgo distribuido.

En el marco de la organización sanitaria, la bibliografía disponible muestra un consenso general acerca de la necesidad de adoptar el liderazgo transformacional en el marco amplio de la humanización de la salud. En esta línea de innovación organizacional, los retos que se imponen son adoptar nuevos roles y responsabilidades profesionales, avanzar hacia nuevas modalidades de planificación e impulsar la participación de todos los agentes implicados. Así mismo, en la literatura disponible se observa la centralidad de tres líneas de investigación claves: la relación del liderazgo con el clima y la satisfacción profesional, el liderazgo enfermero y la formación en el liderazgo.

El panorama de la organización sanitaria actual está, por tanto, orientado hacia estilos de liderazgo descentralizado y participativo, cuyas funciones son asegurar una comunicación óptima dentro de la organización sanitaria y consolidar la motivación, formación y concienciación de los profesionales con respecto a su papel, objetivos y funciones. Futuros estudios realizados en el ámbito de la gestión sanitaria podrán revelar qué estrategias y prácticas garantizarían en mayor medida la consecución de estos objetivos de liderazgo transformacional en el ámbito de la salud. 


\section{Referencias}

Aguilar Luzón, M.C., Calvo Salguero, A., García Hita, M.A., 2007. Valores laborales y percepción del estilo de liderazgo en personal de enfermería. Salud Pública de México, 49(6), 401-7.

Alcázar, F., Otero, A., Iglesias, F., 1996. La figura del coordinador vista por los profesionales del equipo de atención primaria. Cuadernos de Gestión, 2(1), 11-8.

Álvarez de Mon, S., 2006. Liderazgo y dirección de personas: el liderazgo invisible, clave para la sanidad. En: Rosenmöller, M., Ribera, J. y Gutiérrez Fuentes, J.A. (Eds.). Gestión en el sector de la salud (Vol. 2). Madrid: Fundación Lilly, pp. 355-74.

Amabile, T.M., Gryskiewicz, N.D., 1989. The creative environment scales: Work Environment Inventory. Creativity Research Journal, 2, 231-253.

Ángel Jiménez, G., 2006. Concepciones acerca del liderazgo en enfermería. Enfermería Global, 9.

Aronson, E., 2001. Integrating leadership styles and ethical perspectives. Canadian Journal of administrative sciences, 18(4), 244-256.

Arredondo Trapero, F.G., 2002. La integridad en el liderazgo transaccional y transformacional, una aproximación ética. XV Congreso Internacional de Contaduría, Administración e Informática. México, pp. 1-19.

Avolio, B., Walumbwa, F., Weber, T., 2009 Leadership: current theories, research and future directions. Annual Review of Psychology, 60, 421- 49.

Bass, B.M. 1985. Leadership and performance beyond expectations. New York, Free Press.

Bennet, N., Wise, C., Woods, P. et al., 2003. Distributed Leadership. London, NCSL.

Bolívar, A., 2006. A liderança educational e a direcção escolar em Espanha: Entre a necessidade e a (im)posibilidade. Administração Educacional, 6, 76-93.

Büchler, P., Martin, D.F., Büchler, M.W., 2006. Formación en administración de empresas para los cirujanos del futuro: ¿qué nos pueden enseñar las ciencias empresariales? Cirugía española, 79(5), 274-82.

Burke, R. J., Cooper, C. (eds.), 2006. Leading in turbulent times. Oxford, Blackwell.

Burns, J. M., 1978. Leadership. New York, Harper and Row.

Cantera Herrero, F.J., Yáñez, J.L., 2008. Aportaciones del liderazgo positivo (I). Las vitaminas en el trabajo: el bienestar psicológico para retener el talento. Capital humano, 221, 40-8.

Carrasco Acosta, M.C., Márquez Garrido, M., Arenas Fernández, J., 2005. Antropologíaenfermería y perspectiva de género. Cultura de los cuidados, 18, 52-9.

Carson, J.B, Tesluk, P.E., Marrone, J.A., 2007. Shared leadership in teams: An investigation of antecedent conditions and performance. Academy of Management Journal, 50, 12171234.

Chiang Vega, M., Salazar Botello, C., Martín Rodrigo, M. et al., 2011. Clima organizacional y satisfacción laboral: una comparación entre hospitales públicos de alta y baja complejidad. Salud Trab, 19, 1-12.

Chocar, J.S., Brodbeck, F., House, R. J. (Eds.), 2007. Culture and leadership across the world. The GLOBE book of in-depth studies of 25 societies. Mahwah, New Jersey, Lawrance Erlabaum Ass.

Conger, J.A., Kanungo, R.N., 1998. Charismatic Leadership in Organizations. London, Sage. Córdoba Gómez, J., García Vázquez, C., Moreno Domínguez, M.J., 2011. El Servicio Andaluz de Salud apuesta por el aprendizaje. Capital humano, 252, 52-60. 
Day, D.V., Gron, P., Salas, E., 2004. Leadership capacity in teams. The Leadership Quarterly, 857-880.

De Lima Trindade, L. et al., 2011. Influencia de los estilos de liderazgo del enfermero en las relaciones interpersonales del equipo de enfermería. Enfermería global, 22.

Del Llano, J., 2002. Análisis cualitativo de las innovaciones organizativas en hospitales públicos españoles. Gaceta sanitaria, 16(5), 408-416.

Densten, I.L., 2005. The relationship between visioning behaviours of leaders and follower burnout. British Journal of Management, 16, 105-118.

Ekvall, G, Arvonen, J. 1999. Effective leadership: Both universality and contingent? Creativity and Innovation Management, VIII (4), 242-250.

Elenkov, D.S., Manev, I.M., 2009. Senior expatriate leadership's effects on innovation and the role of cultural intelligence. Journal of World Business, 44(4), 357-369.

Ensley, M.D., Hmieleski, K.M., Pearce, C.L., 2006. The importance of vertical and shared leadership within new venture top management teams: Implications for the performance of startups. Leadership Quarterly, 17, 217-231.

Fariña Gómez, B., González González, Y., 1998. Gestión estratégica de la calidad. Herramientas: una aplicación en el campo sanitario. Anales de estudios económicos y empresariales, 13, 275-316.

Fernández Terreros, J., 2006. Entrenamiento en liderazgo y rendimiento de equipos de trabajo. Bilbao: Universidad de País Vasco.

Freire, J., 2011. El buen gobierno, ¿eslabón perdido en la calidad en el SNS español? ReV Calidad Asistencial, 26, 211-4.

García Pérez, M.A., Amaya Pombo, C., 2007. Una sanidad necesitada de liderazgo profesional. Revista de administración sanitaria siglo XXI, 5(2), 275-282.

Gil, F., Alcover, C. M., Rico, R., Sánchez-Manzanares, M., 2011. Nuevas formas de liderazgo en equipos de trabajo. Papeles del Psicólogo, 32(1), 38-47.

Goetz, K., Beutel, S., Mahler, C. et al., 2012. Comportamiento relacionado con el trabajo y patrones de experiencia de enfermeras. INR, 1, 96-102.

González, S., Beltri, F., Rubi, A., 2005. Liderazgo de libertades: libertazgo. Capital Humano, 186, 54-61.

Greenleaf, R.K., 1997. Servant leadership: a journey into the nature of legitimate greatness. Mahwah, Paulist Press.

Hackman, J.R., Wagerman, R., 2005. A theory of team coaching. Academy of Management Review, 30, 269-287.

Harms, P. D., Crede, M. 2010. Emotional intelligence and transformational and transactional leadership: a meta-analysis. Journal of Leadership and Organizational Studies, 1, 5-17.

Heifetz, R.A., 1994. Leadership without Easy Answers. Cambridge, Belknap Press.

House, R., Aditya, R., 1997. The Social Scientific Study of Leadership: Quo Vadis?. Journal of Management, 23(3), 409-473.

Hoyos Hernández, P.A., Cardona Ramírez, M.A., Correa Sánchez, D., 2008. Humanizar los contextos de salud, cuestión de liderazgo. Investigación y educación en enfermería, 26(2), 218-25.

Huang, R., Kahai, S., Jestice, R., 2010. The contingent effects of leadership on team collaboration in virtual teams. Computers in Human Behavior, 26, 1098-1110.

Janssen, O., De Vliert, E., West, M., 2004. The bright and dark sides of individual and group innovation: a Special Issue introduction. Journal of Organizational Behavior, 25(2), 129-45. 
Judge, T.A., Piccolo, R.F., 2004. Transformational and transactional leadership: a metaanalytic test of their relative validity. Journal of Applied Psychology; 89(5), 755-68.

Kaufmann, A.E. 1997. Liderazgo transformador y formación continua. REIS, 77, 163-184.

Keeley, M. 1998. The trouble with transformational leadership: toward a federalist ethic for organizations. En: Ciulla, JB (Ed.), Ethics the heart of leadership. Wesport, CT, Praeger.

Kelloway, K., Loughlin, C., Barling, J. et al., 2002. Counterproductive and organizational citizenship behaviours: separate but related constructs. International Journal of Selection and Assessment, 10(1-2), 143-151.

Koch, H., 1994. Gestión Total de la Calidad en la Sanidad. Barcelona, SG editores.

Lauzon, S., Ricard, N., Goulet, C., 2003. Enfermería de práctica avanzada: un tesoro oculto. Enfermería clínica, 13(1), 48-52.

Linley, P.A., Joseph, S., Seligman, M.E.P., 2004. Positive psychology in practice. New Jersey, John Wiley \& Sons.

Marion, R, Uhl-Bien, M., 2007. Introduction to the special issue on leadership and complexity. The Leadership Quarterly, 18, 293-6.

Martin, D.R, o'Brien. J.L., Heyworth, J. A. et al. 2008. Point counter- point: the function of contradictions on an interdisciplinary health care team. Qual Health Res, 18, 369-79.

Mehra, A., Smith, B., Dixon, A., Robertson, B. 2006. Distributed leadership in teams: The network of leader- ship perceptions and team performance. Leadership Quarterly, 17, 232245.

Menárguez Puche, J., Saturno Hernánde, P. 1998. Características del liderazgo de los coordinadores de centros de salud en la Comunidad Autónoma de Murcia. Aten Primaria, 22(10), 636-41.

Michie, S., Gootie, J. 2005. Values, emotions and authenticity: Will the real leader please stand up? The Leadership Quarterly, 16, 441-57.

Molero Alonso, F. 2002. Cultura y liderazgo. Una relación multifacética. Boletín de Psicología, 76, 53-76.

Morales, J.F., Molero, F., 1995. El liderazgo en los equipos de atención primaria. Cuadernos de Gestión para el Profesional de Atención Primaria, 1(2), 83-91.

Morán Barrios, J., Ruiz de Gauna, P., 2010. ¿Reinventar la formación de médicos especialistas?: Principios y retos. Nefrología, 30(6), 604-612.

Moreno Pérez, C.M., 2004. Claves para el liderazgo ético. Capital Humano, 183, 84-9.

Morgueson, F.P., Scott, D., Karam, E.P., 2010. Leadership in teams: a functional approach to understanding leadership structures and processes. Journal of Management, 36(1), 5-39.

Pearce, C.L., Conger, J.A., 2003. Shared leadership: reframing the hows and whys of leadership. Thousand Oaks, CA, Sage.

Peiró, J. +M., González-Roma, V., Ramos, J., Zornoza, A., 1996. Relationships between leadership and professionals' job attitudes and perceptions: comparison of two leadership models. Work \& Stress, 10(3), 195-208.

Peiró, J.M., González-Romá, V., Ripoll, P., Gracia, F., (2001). Role stress and work team variables in Primary Health Care Teams. In: De Jonge $\mathrm{J}$ et al (eds). Organizational Psychology and Health Care at the Start of a New Millennium, Munich: Rainer Hampp Verlog, pp 105-22.

Peiró, J.M., Rodríguez, I., 2008. Estrés laboral, liderazgo y salud organizacional. Papeles del Psicólogo, 29(1), 68-82. 
Peya Gascóns, M., 2008. La importancia del liderazgo formal en la profesión enfermera. Nursing, 26.

Pons Verdú, F., Ramos López, J., 2012. Influencia de los Estilos de Liderazgo y las Prácticas de Gestión de RRHH sobre el Clima Organizacional de Innovación. Revista de psicología del Trabajo y de las Organizaciones, 28(2), 81-98.

Rico, R., Alcover De La Hera, C.M., Tabernero, C., 2010. Efectividad de los Equipos de Trabajo, una Revisión de la Última Década de Investigación (1999-2009). Revista de Psicología del Trabajo y de las Organizaciones, 26(1), 47-71.

Robles-García, M., Dierssen-Sotos, T., Martínez-Ochoa, E. et al., 2005. Variables relacionadas con la satisfacción laboral: un estudio transversal a partir del modelo EFQM. Gac Sanit, 19(4), 127-34.

Ruiz López, P., Carrasco González, I., 2010. Liderazgo en la seguridad del paciente. Rev Calidad Asistencial, 25, 55-7.

Salas Vallina, A., 2013. Liderazgo transformacional, capacidad de aprendizaje organizativo y felicidad en el trabajo. Tesis doctoral. Universidad de Valencia.

Sanders, J.O., 1995. Liderazgo espiritual. Grand Rapids, Editorial Portavoz.

Sashkin, M., 1998. El líder visionario: Cuestionario sobre la conducta del líder. Madrid, Editorial Centro de Estudios Ramón Areces.

Senge, P.M., 1990. The Fifth Discipline: The Art and Practice of the Learning Organization. New York, Doubleday/Currency.

Shamir, B., House, R.J., Arthur, M.B., 1993. The motivational effects of charismatic leadership: A self-concept based theory, Organization Science, 4, 1-17.

Sosik, J.J., Godshalk, V.M., 2000. Leadership styles, mentoring functions received and jobrelated stress: a conceptual model and preliminary results. Journal of Organizational Behavior, 21(4), 365-390.

Souza, L., Ferreira, A., Nunes, R., Maxwell de Freitas, J., Veloso, O., 2013. El liderazgo en la visión de enfermeros líderes. Enfermería Global, 12(2), 268-80.

Spillane, J.P., Diamond, J.B. (Eds.), (2007. Distributed leadership in practice. New York, Teachers College Press.

Stewart, G.L., 2006. A meta-analytic review of relation- ships between team design features and team performance. Journal of Management, 31, 319-335.

Stoker, J.I., Looise, J.C., Fisscher, O.A.M., de Jong, R.D., 2001. Leadership and innovation: relations between leadership, individual characteristics and the functioning of $R$ \& $D$ teams. International Journal of Human Resource Management, 141-1151.

Subirana, M., Fargues, I., 2005. Percepción de las enfermeras expertas sobre el reconocimiento de sus competencias. Metas de Enfermería, 8, 62-8.

Teixidor i Freixa M., 2004. Ya en el siglo XXI... Un nuevo liderazgo en ciencias de la salud. Revista ROL de enfermería, 27, 70-2.

Torrado, J., 2012. Liderazgo entre iguales en equipos deportivos: una revisión camino a la integración. Escritos de Psicología, 5(2), 12-19.

Tresserras i Gaju, R., 2008. Planificación según prioridades de salud: Criterios de planificación relacionados con los Planes Directores. Medicina clínica, 131(4), 42-6.

Trice, H.M., Beyer, J.M., 1993. The cultures of work organization. Englewood Cliffs, Prentice Hall.

Wageman, R., 2001. How leaders foster self-managing team effectiveness: Design choices versus hands-on coaching. Organization Science, 12, 559-577. 
Yukl, G.A., 1998. Leadership in organizations. Englewood Cliffs, N.J, Prentice Hall.

Yukl, G.A., 2004. Tridimensional leaderchip theory: a road-map for flexible, adaptative leaders. En: Burke R (Ed.), Leading in turbulent times. Oxford, Blackwell, pp. 75-91.

Zaccaro, S., Rittman, A., Marks, M.A., 2001. Team Leadership. The Leadership Quarterly, $12,451-83$. 